\title{
Editorial
}

\section{Functional Nanomaterials for Electronics, Optoelectronics, and Bioelectronics}

\author{
Neeraj Dwivedi, ${ }^{1}$ Sushil Kumar, ${ }^{2}$ J. David Carey, ${ }^{3,4}$ and Chetna Dhand ${ }^{5}$ \\ ${ }^{1}$ Department of Electrical and Computer Engineering, National University of Singapore, Singapore \\ ${ }^{2}$ CSIR-National Physical Laboratory, New Delhi, India \\ ${ }^{3}$ Advanced Technology Institute, University of Surrey, Guildford, Surrey, UK \\ ${ }^{4}$ Department of Electronic Engineering, University of Surrey, Guildford, Surrey, UK \\ ${ }^{5}$ Singapore Eye Research Institute, Singapore 169856 \\ Correspondence should be addressed to Neeraj Dwivedi; neerajdwivedi6@gmail.com
}

Received 19 May 2015; Accepted 26 May 2015

Copyright (C) 2015 Neeraj Dwivedi et al. This is an open access article distributed under the Creative Commons Attribution License, which permits unrestricted use, distribution, and reproduction in any medium, provided the original work is properly cited.

With the miniaturization of electronic devices and advances in nanomaterial research and production, the application of functional nanomaterials is at the forefront of scientific and industrial attention. The use of nanomaterials on their own, or as part of a hybrid structure, allows new properties to be exploited in the areas of electronics, optoelectronics and photonics, and in bioelectronics. Recent advances in carbon nanomaterials, such as nanotubes and graphene, as well as other 2D layered materials, in nanostructured organic conducting polymers and in metal nanoparticles have broadened the scope of nanomaterials towards innovation in electronic and bioelectronic devices. This innovation has the potential to develop new consumer electronics, energy generation and storage technologies, and information communications and technology and in the areas of medical diagnosis and treatment.

This special issue is focused on utilization of functional nanomaterials for electronics, optoelectronics, and bioelectronics.

W.-K. Hong and colleagues have systematically compiled an important review on the study of the metal-insulator phase transition in quasi-one-dimensional $\mathrm{VO}_{2}$ structures. They discuss tailoring the metal-insulator transition by various approaches which can be employed for gas sensor, strain sensor, switching, and memory applications.

Improved photovoltage and photocurrent density is reported by C. A. Rodríguez-Castañeda et al. in their study of microwave synthesized monodisperse CdS spheres of different size and color for solar cell applications. They have synthesized CdS spheres of sizes from $40-50 \mathrm{~nm}$ to $140 \mathrm{~nm}$ which when mixed into $\mathrm{P} 3 \mathrm{HT}$ result in improved photoactive response. Staying with photoactive materials, J. S. Lee et al. report the enhancement of dye synthesized solar cell performance by the doping of $\mathrm{TiCl}_{4} / \mathrm{Al}_{2} \mathrm{O}_{3}$ in $\mathrm{TiO}_{2}$ nanotube array photoelectrodes.

G. Wang et al. report theoretical calculations of the effects of barrier thickness and hydrostatic pressure on hydrogenic impurity wurtzite $\mathrm{GaN} / \mathrm{Al}_{x} \mathrm{Ga}_{1-x} \mathrm{~N}$ strained quantum dots and discuss the potential for advancing the applications of group III nitride materials. In the work by A. Kelchtermans et al., ways to increase the solubility limit of tetrahedral $\mathrm{Al}$ in $\mathrm{ZnO}: \mathrm{Al}$ nanorods are discussed. By using the optimum parameters they have enhanced the substitution of $\mathrm{Al}$ in $\mathrm{ZnO}: \mathrm{Al}$, which is useful for enhancing its conductivity and other properties.

S.-J. Hwang et al. report their research on the fabrication of liquid crystal (LC) microlens using nanoparticle-induced vertical alignment. The nanoparticle-induced vertical alignment of the nematic liquid crystals is applied to achieve an adaptive flat LC microlens with a hybrid-aligned nematic mode by dropping polyhedral oligomeric silsesquioxane nanoparticle solution on a homogeneous alignment layer.

Finally, F. Severiano et al. have reported their work on the importance of the electrolyte in obtaining porous silicon and how it modifies the optical and structural proprieties.

All of these papers show how nanomaterials can have a significant benefit in a wide range of materials and device research. It is hoped that readers of this special issue will 
see a snapshot of the breath of current research in functional materials science and engineering.

\section{Acknowledgments}

The editors would like to thank the authors who have submitted their research to this special issue. The editors also acknowledge all reviewers for their contribution to this special issue. The lead guest editor thanks all guest editors for spending their precious time in handling the paper.

Neeraj Dwivedi Sushil Kumar J. David Carey Chetna Dhand 

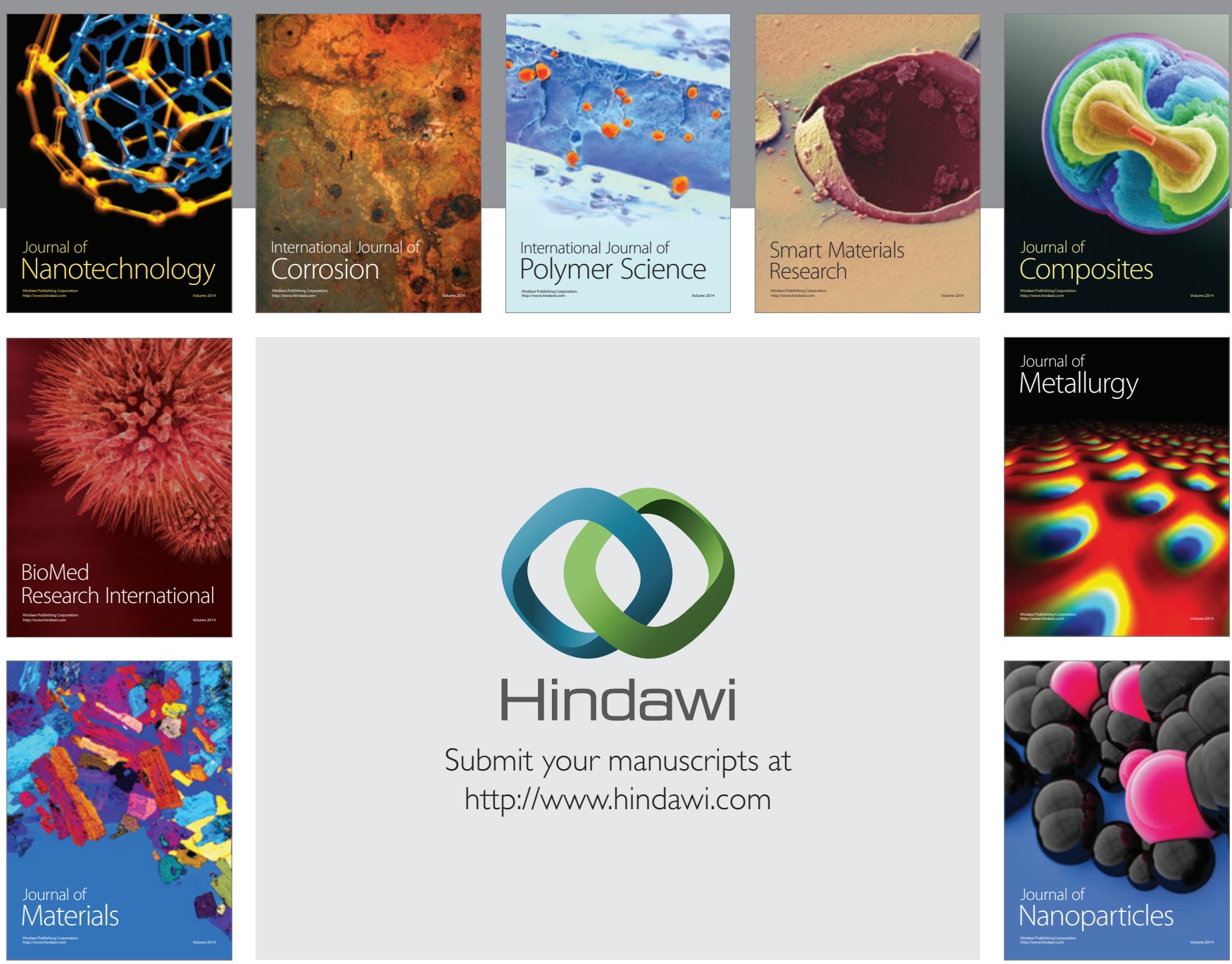

Submit your manuscripts at http://www.hindawi.com
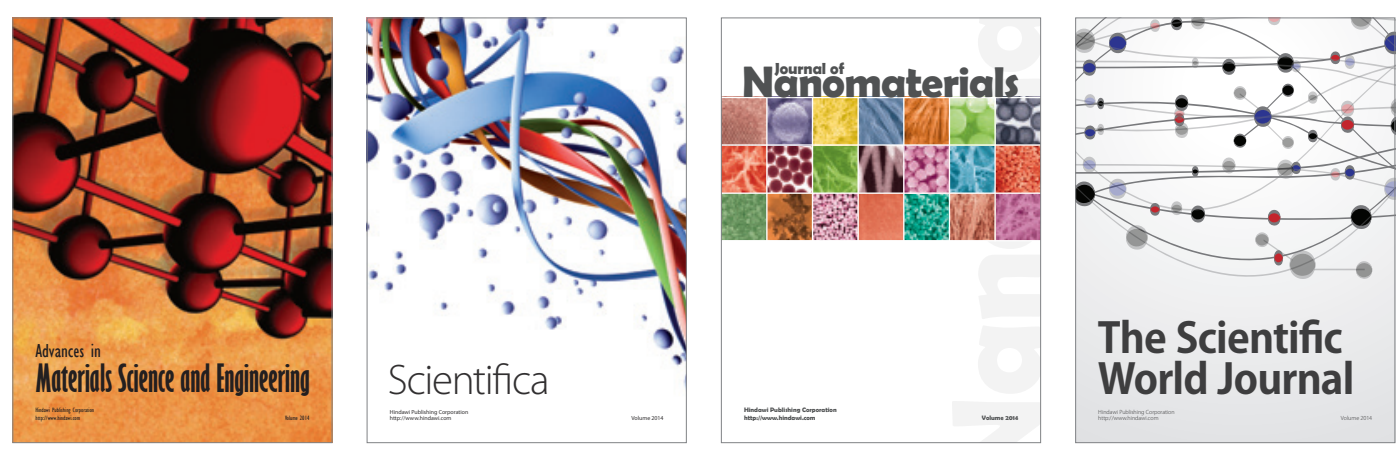

\section{The Scientific World Journal}
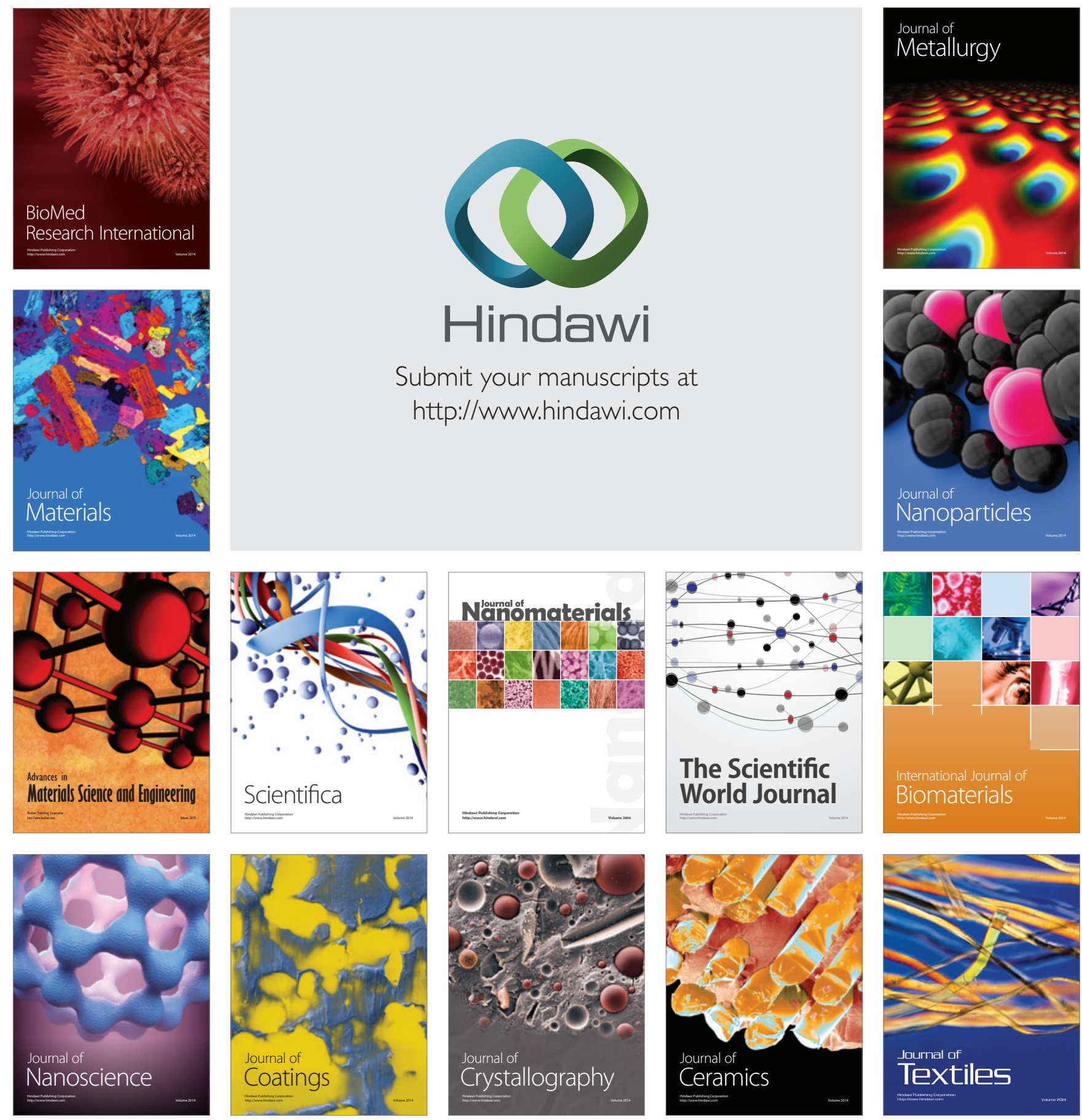